\title{
Reasons for requesting removal of the hormonal implant, Implanon NXT, at an urban reproductive health clinic in KwaZulu-Natal, South Africa
}

\author{
I Beesham, ${ }^{1} \mathrm{MB}$ ChB; J Smit, ${ }^{1} \mathrm{PhD}$; M Beksinska, ${ }^{1} \mathrm{PhD}$; M Panday, ${ }^{2} \mathrm{MB}$ ChB, FCOG (SA); V Makatini, ${ }^{3} \mathrm{RNMSN}, \mathrm{MPH} ; \mathrm{S}$ Evans, ${ }^{1} \mathrm{MB}$ ChB \\ ${ }^{1}$ MatCH Research Unit (MRU), Faculty of Health Sciences, University of the Witwatersrand, Johannesburg, South Africa \\ ${ }^{2}$ King Dinuzulu Hospital Complex, KwaZulu-Natal Provincial Department of Health, Durban, South Africa \\ ${ }^{3}$ KwaZulu-Natal Provincial Department of Health, Durban, South Africa
}

Corresponding author: I Beesham (ibeesham@mru.ac.za)

Background. The contraceptive implant, Implanon NXT, was introduced in South Africa (SA) in 2014 and, although it offers multiple advantages, users may request to have it removed early for several reasons. The number of insertions of Implanon NXT has declined in SA and there have been concerns about early removals.

Objectives. To gain an understanding of patterns of Implanon NXT use, reasons for requesting removal and duration of use at the time of requesting removal.

Methods. This was a cross-sectional study conducted at an urban public-sector reproductive health clinic in the eThekwini District of KwaZulu-Natal, SA. A total of 120 women $\geq 18$ years of age requesting removal of Implanon NXT completed an interviewer-administered questionnaire that probed experiences of use and reasons for removal. Data were collected electronically on Wits REDCap (Research Electronic Data Capture) and analysed using Stata 14 (StataCorp, USA). The study was conducted from 2017 to 2018.

Results. A total of 120 women were interviewed. Their mean age was 28 (range $19-44)$ years and most women $(n=103 ; 85.8 \%)$ had completed secondary school. The majority were black $(n=115 ; 95.8 \%)$ and unmarried $(n=102 ; 85 \%)$. Implants had been inserted primarily by nurses $(n=110 ; 91.7 \%)$ at public-sector clinics $(n=91 ; 75.8 \%)$. Three-quarters of the women $(n=91 ; 75.8 \%)$ requested removal of Implanon NXT because it had reached the intended 3-year duration. Reasons for early removal were mainly due to side-effects, e.g. bleeding problems ( $n=19 ; 15.8 \%)$, weight gain $(n=7 ; 5.8 \%)$, loss of libido $(n=2 ; 1.7 \%)$, headaches $(n=5 ; 4.2 \%)$, dizziness $(n=4 ; 3.3 \%)$ and pain/numbness in the $\operatorname{arm}(n=2 ; 1.7 \%)$. Just more than half $(57.1 \%)$ of the women who had received the implant for the intended 3-year duration had requested reinsertion of Implanon NXT.

Conclusions. The main reason for requesting removal was that Implanon NXT had reached its intended 3-year duration, and more than half of the women requested reinsertion of the device following removal. Implanon NXT is a highly effective, safe, acceptable, long-acting contraceptive and important in the SA contraceptive method mix.

S Afr Med J 2019;109(10):750-755. https://doi.org/10.7196/SAMJ.2019.v109i10.12

Implanon NXT is a single-rod progestin-only implant that contains $68 \mathrm{mg}$ of etonogestrel preloaded in a disposable applicator. ${ }^{[1]}$ It is indicated for use by women to prevent pregnancy. Implanon NXT was first launched in Indonesia in $1998^{[2]}$ and was approved by the US Food and Drug Administration in 2006. ${ }^{[3]}$ Implants belong to a class of long-acting reversible contraceptives and along with intrauterine devices (IUDs) are referred to as the 'most effective methods of reversible contraception. ${ }^{[4]}$ In 2012, the United Nations Commission on Life-Saving Commodities for Women and Children ${ }^{[4]}$ endorsed contraceptive implants as one of its 13 life-saving commodities.

According to the South African Demographic and Health Survey (SADHS) 2016, 58\% of women (those currently married, and those sexually active and unmarried) use a modern contraceptive method, but $18 \%$ of women continue to have an unmet family planning need. ${ }^{[5]}$ The SA National Contraceptive and Fertility Planning Policy aims to expand access to long-acting contraceptive methods. ${ }^{[6]}$ The National Department of Health (NDoH) introduced Implanon NXT in 2014. In that year, insertions peaked in SA, with $>175000$ insertions in the 2014/2015 reporting period, but this declined rapidly, with a $50 \%$ decrease between 2014/2015 and 2015/2016, ${ }^{[7]}$ and a 73\% drop between 2014/2015 and 2016/2017. ${ }^{[8]}$ The decline might be attributed to the pool of women interested in this method being saturated after the initial peak, with insertions continuing to stabilise at lower levels. Other explanations could include negative media reports ${ }^{[9-11]}$ and community awareness of the side-effects. Another consideration is the release of a circular in October 2014 by the $\mathrm{NDoH}$, recommending that women on enzyme-inducing drugs should not use the implant, but rather an alternative method, and those already on the implant should be given the option of removal. ${ }^{[12]}$ Consequently, many antiretroviral (ARV) clinics across the country abruptly stopped providing Implanon NXT. ${ }^{[13]}$

Studies found that women may request early removal of the implant for a variety of reasons. Some of the initial international studies on Implanon NXT found that bleeding irregularities and other adverse events were common reasons for early removal. ${ }^{[14-16]}$ Bleeding disturbances were the most frequent reason for discontinuation of implants in a large multicentre study in 7 countries. ${ }^{[17]}$ Other adverse events leading to discontinuation included weight gain, acne, emotional lability and headaches. ${ }^{[14]}$ A limited number of studies in SA have investigated discontinuation of the implant. A study conducted in the Eastern Cape found that side-effects such as heavy bleeding, severe headache and painful arm were the main reasons for 
early Implanon NXT removal - the mean duration of use was 11.2 months ${ }^{[18]}$ Another study conducted in 2 provinces in SA found that $90 \%$ of removals were due to side-effects and two-thirds related to changed bleeding patterns. ${ }^{[19]}$ One study conducted at a hospital in KwaZulu-Natal (KZN) found that $\sim 50 \%$ of Implanon NXT removals were due to abnormal uterine bleeding. ${ }^{[13]}$

Users may also have the implant removed for reasons other than sideeffects. Studies indicated that reasons such as the desire to conceive, receiving other medical treatment (e.g. ARVs), misinformation and rumours, planning to go abroad and inadequate counselling might also result in early removal. ${ }^{[18,19]}$ A local study conducted among nurses found that they lacked confidence in providing implant services effectively and generally held negative views towards the method, but the study was limited by its sample size. ${ }^{[20]}$

Reasons for implant removal are not well reported in SA and there are limited data from KZN, which has the highest recorded uptake of Implanon NXT. ${ }^{[5]}$ This study aimed to look at the reasons for requesting removal of Implanon NXT in an urban reproductive health clinic $\sim 3$ years after its introduction in SA, amid declining rates of insertions and concerns about early removals.

\section{Methods}

A descriptive cross-sectional study among Implanon NXT users who requested removal was conducted at an urban public-sector reproductive health clinic in the eThekwini District of KZN, where $\sim 6000$ women attend per month. A range of services are provided at no cost and include HIV counselling and testing, initiation and management of patients on ARVs, cervical cancer screening, diagnosis and management of sexually transmitted infections, family planning services and assessment and management of gynaecology patients. The clinic also provides contraceptive training (including implant insertions and removals) to medical and nursing students, as well as doctors and nurses. Women attending this clinic include students in higher education, and urban and peri-urban women (employed and unemployed). The clinic is centrally located and provides services to a diverse population of women who live and work in the city centre, but also to many women from surrounding urban and peri-urban areas - up to $30 \mathrm{~km}$ away.

A total of 120 women were interviewed from December 2017 to April 2018 using consecutive sampling. Interviews were conducted in English or isiZulu, depending on participant preference. DoH clinic staff were introduced to the study and were asked to refer all women requesting Implanon NXT removal to study staff, who provided them with an information sheet with regard to the study.

To be eligible for the study, women had to be $\geq 18$ years old, should have requested removal of the Implanon NXT device or have had the implant removed on the day of the interview, should have documented proof of the family planning card requesting removal or of having had the Implanon NXT removed, should have been willing to provide informed consent and must be either English or isiZulu speaking. Written informed consent was obtained. Women were reimbursed ZAR70 for their time.

Data were collected by trained study staff and included demographics, reproductive history and previous contraceptive use. Women were asked where they had first heard about Implanon NXT, who inserted it and at which facility, bleeding pattern while on Implanon NXT, side-effects experienced and whether they would consider having the implant reinserted in the future. We also asked questions relating to reasons for requesting removal of the implant and the next method the woman intended to use after removal. Early removals were defined as those prior to 3 years from the date of insertion. Data were collected electronically on Wits REDCap (Research Electronic Data Capture), using tablets. Data were analysed using Stata 14 (StataCorp, USA). ${ }^{[21]}$

\section{Ethical approval}

The study was approved by the Human Research Ethics Committee, University of the Witwatersrand, Johannesburg, SA (ref. no. 170109), and by the KZN Provincial DoH (ref. no. HRKM 158/17). A letter of support was obtained from the public-sector clinic where the study was conducted.

\section{Results}

A total of 120 women requesting removal of Implanon NXT were interviewed from December 2017 to April 2018. Their mean age was 28 (range $19-44)$ years. Almost a third $(n=38 ; 31.7 \%)$ of users were between the ages of 18 and 24 years. Most were black ( $n=115$; $95.8 \%)$ and had completed secondary school $(n=103 ; 85.8 \%)$. Half of the women were employed and two-thirds $(n=83 ; 69.2 \%)$ were unmarried and did not live with their current partner.

Most women $(n=97 ; 80.8 \%)$ reported ever being pregnant and a third $(n=40 ; 33.3 \%)$ planned to have children in the future. About a fifth were nulliparous. When asked about previous contraceptive use, half $(n=67 ; 55.8 \%)$ had used the 3-month injectable method, 27 (22.5\%) used the 2-month injectable method, and $85(70.8 \%)$ had used male condoms. None had used the IUD previously. Five (4.2\%) women had previously used Implanon NXT. Table 1 provides study participant characteristics.

Almost half of the women had heard about the implant from clinic staff $(n=57 ; 47.5 \%)$ or a friend or relative $(n=53 ; 44.2 \%)$. Implant insertions had been carried out primarily by nurses $(n=110$; $91.2 \%)$ and at public-sector clinics ( $n=91 ; 75.8 \%)$. About a quarter of insertions $(n=27 ; 22.5 \%)$ were carried out at government hospitals. Only 49 (40.8\%) implants were inserted at the clinic where the study was conducted. Two-thirds $(n=77 ; 64.2 \%)$ of the women chose to use Implanon NXT, as it was a long-acting method, and half ( $n=60$; $50 \%$ ) because they did not want to return to the clinic frequently for follow-up.

Women were asked regarding counselling or information provided prior to insertion. Most reported being counselled on how long the implant lasts $(n=103 ; 86.6 \%)$ and when to return for removal $(n=105$; $88.2 \%)$. Ninety-three (78.2\%) women were counselled on possible side-effects, two-thirds $(n=79 ; 66.4 \%)$ recalled being counselled on the advantages of the implant, and only 9 (7.6\%) could not remember what counselling they had received before insertion of the implant.

Three-quarters ( $n=91 ; 75.8 \%$ ) of women had kept the implant for a duration of $\geq 3$ years. The remaining quarter had requested removal of the implant before 3 years (Table 2).

The main reason for requesting Implanon NXT removal $(n=91$; $75.8 \%$ ) was because it had reached its intended 3-year duration. Women could select multiple responses as reasons for requesting removal of the implant. From the 29 early removals, 24 (82.8\%) women mentioned side-effects as a reason. Bleeding was the most common reason for early removal of the implant $(n=19 ; 15.8 \%)$, with 14 women reporting prolonged or heavy bleeding and 10 reporting irregular bleeding. Other side-effects leading to early Implanon NXT removal were weight gain $(n=7 ; 5.8 \%)$, loss of libido $(n=2 ; 1.7 \%)$, headaches $(n=5 ; 4.2 \%)$, dizziness $(n=4 ; 3.3 \%)$ and pain/numbness in the $\operatorname{arm}(n=2 ; 1.7 \%)$.

Five women had requested early implant removal for reasons other than side-effects -2 of them had reported being pregnant or wanting to conceive. One woman had the implant removed at her partner's 


\section{Table 1. Characteristics of study participants}

\begin{tabular}{|c|c|}
\hline Characteristics & $n(\%)(n=120)$ \\
\hline \multicolumn{2}{|l|}{ Age, years } \\
\hline $18-24$ & $38(31.7)$ \\
\hline $25-30$ & $41(34.2)$ \\
\hline $31-35$ & $26(21.7)$ \\
\hline $36-40$ & $12(10)$ \\
\hline$>40$ & $3(2.5)$ \\
\hline \multicolumn{2}{|l|}{ Race } \\
\hline Black & $115(95.8)$ \\
\hline White & $1(0.8)$ \\
\hline Coloured & $2(1.7)$ \\
\hline Indian & $1(0.8)$ \\
\hline Other & $1(0.8)$ \\
\hline \multicolumn{2}{|l|}{ Highest level of education } \\
\hline Secondary school (incomplete) & $17(14.2)$ \\
\hline Secondary school (complete) & $35(29.2)$ \\
\hline Tertiary/college/university (incomplete) & $28(23.3)$ \\
\hline Tertiary/college/university (complete) & $40(33.3)$ \\
\hline \multicolumn{2}{|l|}{ Employed } \\
\hline Yes & $60(50.0)$ \\
\hline No & $60(50.0)$ \\
\hline \multicolumn{2}{|l|}{ Relationship with partner } \\
\hline Married and living together & $13(10.8)$ \\
\hline Married and not living together & $5(4.2)$ \\
\hline Not married and living together & $19(15.8)$ \\
\hline Not married and not living together & $83(69.2)$ \\
\hline \multicolumn{2}{|l|}{ Ever pregnant } \\
\hline Yes & $97(80.8)$ \\
\hline \multicolumn{2}{|l|}{ Pregnancies, $n$} \\
\hline 0 & $23(19.2)$ \\
\hline $1-2$ & $74(61.7)$ \\
\hline$\geq 3$ & $23(19.2)$ \\
\hline \multicolumn{2}{|l|}{ Living children, $n$} \\
\hline 0 & $2(1.7)$ \\
\hline $1-2$ & $78(65)$ \\
\hline$\geq 3$ & $17(14.2)$ \\
\hline \multicolumn{2}{|l|}{ Plan on having more children } \\
\hline Yes & $40(33.3)$ \\
\hline No & $62(51.2)$ \\
\hline Maybe & $18(15)$ \\
\hline \multicolumn{2}{|l|}{ Contraceptive method ever used } \\
\hline Male condoms & $85(70.8)$ \\
\hline Female condoms & $3(2.5)$ \\
\hline 3-month injection & $67(55.8)$ \\
\hline 2-month injection & $27(22.5)$ \\
\hline Oral contraceptive & $23(19.2)$ \\
\hline Implanon NXT & $5(4.2)$ \\
\hline Emergency contraception & $2(1.7)$ \\
\hline Natural methods, e.g. withdrawal & 0 \\
\hline Intrauterine device & 0 \\
\hline None & $7(5.8)$ \\
\hline
\end{tabular}

request (religious reasons), 1 implant was removed at the provider's suggestion owing to concern regarding drug interaction between antiretroviral treatment (ART) and the implant, and the fifth removal was at the request of the participant owing to relocation and concern about finding a facility where it could be removed.
Only 4 (3.3\%) women requested removal of the implant (both early and at 3 years) because of pregnancy or wanting to conceive. Two cases of pregnancy were reported while the women were on Implanon NXT 1 woman had been on the implant beyond the intended 3-year duration (3 years and 10 months), and her timing of conception was calculated 
to be $\sim 6$ months after her implant had reached its 3 -year duration. The other participant reported a positive home urine pregnancy test, but was awaiting confirmation of pregnancy by ultrasound examination, as the clinic urine pregnancy test was negative. On further follow-up, this participant was confirmed to be pregnant. Table 3 lists reasons for requesting removal of Implanon NXT.

The 5 women who reported previous Implanon NXT use had it removed early ( $\leq 12$ months) after the second insertion. Four of these women requested early removal owing to side-effects, and 3 of these had experienced no problems with the initial implant. Table 4 lists reasons for removal of Implanon NXT in women who had used it previously.

Over half ( $n=52 ; 57.1 \%$ ) of the 91 women who had been using the implant for 3 years chose to reinsert it after removal. Of the remaining 39 women, 6 (6.6\%) chose male condoms, $13(14.3 \%)$ injectables, $5(5.5 \%)$ an IUD, $5(5.5 \%)$ oral contraceptives, $2(2.2 \%)$ tubal ligation,

Table 2. Duration of Implanon NXT use at the time of requesting removal

\begin{tabular}{ll}
\hline Duration & $\boldsymbol{n}(\%)(\boldsymbol{n}=\mathbf{1 2 0})$ \\
\hline$<6$ months & $9(7.5)$ \\
6 months - 1 year & $4(3.3)$ \\
$>1$ year, $<2$ years & $6(5)$ \\
$\geq 2$ years, $<3$ years & $10(8.3)$ \\
3 years & $91(75.8)$
\end{tabular}

Table 3. Reasons for requesting removal of Implanon NXT

\begin{tabular}{ll}
\hline Reason & $\boldsymbol{n}(\%)(\boldsymbol{n}=\mathbf{1 2 0})$ \\
\hline Reached intended 3-year duration & $91(75.8)$ \\
Problems with bleeding & $19(15.8)$ \\
Weight gain & $7(5.8)$ \\
Headaches & $5(4.2)$ \\
Dizziness & $4(3.3)$ \\
Loss of libido & $2(1.7)$ \\
Pain/numbness in the arm & $2(1.7)$ \\
Pregnancy $/$ /want to conceive & $4(3.3)$ \\
Other & $3(2.5)$ \\
*Two cases of confirmed pregnancy were reported on Implanon NXT. One woman was \\
on Implanon NXT beyond the intended 3-year duration. The other woman reported a \\
positive urine pregnancy test done at home, but a urine pregnancy test done at the clinic \\
was negative. She was waiting for an ultrasound examination, and was confirmed pregnant \\
on follow-up.
\end{tabular}

and 7 (7.7\%) no method. Table 5 shows the contraceptive method chosen by women who had been on Implanon NXT for 3 years. Of the 7 women who chose to use no method, 3 wished to conceive, 1 was pregnant, 2 did not desire contraception (not sexually active) and 1 wanted her menses to return before choosing her next contraceptive method. Hence, just over a third of women $(n=31 ; 34.1 \%)$ elected to use a different contraceptive method after keeping Implanon NXT for its intended 3-year duration.

More than half of the women $(n=73 ; 60.8 \%)$ reported that they would be willing to insert Implanon NXT in the future.

\section{Discussion}

This study provides important information and useful insights into patterns of Implanon NXT use and reasons for requesting removal among users in KZN. The study was conducted $\sim 3$ years after the introduction of Implanon NXT in SA and therefore provides an important indication about trends in usage, as all users requesting removal were interviewed and not only those requesting early removal. It is also a time when one would expect public awareness and use to have stabilised and provider skills to be optimised.

We found high levels of satisfaction regarding the implant and three-quarters of the women remained on Implanon NXT for 3 years. They had requested removal, as the implant had reached its intended duration of use. Side-effects accounted for the majority of early removals, and the most common side-effect leading to early removal was bleeding. This has been found in several other studies; ${ }^{[14-17]}$ bleeding changes are expected with Implanon NXT use. Similar findings were found in another SA study, where $90 \%$ of early

Table 5. Contraceptive method chosen after Implanon NXT removal in users who had been on the implant for 3 years

\begin{tabular}{ll}
\hline Contraceptive method & $\boldsymbol{n}(\mathbf{\%})(\boldsymbol{n}=\mathbf{9 1})$ \\
\hline Implanon NXT & $52(57.1)$ \\
Male condoms & $6(6.6)$ \\
2-month injection & $6(6.6)$ \\
3-month injection & $7(7.7)$ \\
Intrauterine device & $5(5.5)$ \\
Oral contraceptives & $5(5.5)$ \\
Tubal ligation & $2(2.2)$ \\
Unsure & $1(1.1)$ \\
No method & $7(7.7)$
\end{tabular}

Table 4. Reasons for removal of Implanon NXT in women who had used it previously

\begin{tabular}{|c|c|c|}
\hline Participant & $\begin{array}{l}\text { Duration of use of second } \\
\text { Implanon NXT, months }\end{array}$ & Reasons for requesting removal of second Implanon NXT \\
\hline \multirow[t]{2}{*}{1} & 6 & Weight gain, loss of libido \\
\hline & & Loss of libido since the first implant insertion \\
\hline \multirow[t]{3}{*}{2} & 4 & 'Pregnancy symptoms', but pregnancy tests had been negative \\
\hline & & Weight gain, dizziness and pain/numbness in the arm \\
\hline & & No side-effects with the first implant \\
\hline \multirow[t]{3}{*}{3} & 12 & Weight gain, problems with bleeding and prolonged nausea \\
\hline & & Nausea daily since insertion and patient suspected pregnancy \\
\hline & & No side-effects with the first implant \\
\hline \multirow[t]{3}{*}{4} & 6 & Home pregnancy test was positive, but clinic urine pregnancy test was negative \\
\hline & & Patient wanted implant removed because, if she was pregnant, it might affect the baby \\
\hline & & On follow-up, confirmation of pregnancy \\
\hline \multirow[t]{2}{*}{5} & 11 & Problems with bleeding (prolonged/heavy bleeding) \\
\hline & & No side-effects with the first implant \\
\hline
\end{tabular}


removals were due to side-effects and two-thirds were related to changes in bleeding patterns. ${ }^{[19]}$ This highlights the importance of counselling women about potential side-effects and how to manage them before providing the method. Few women reported weight gain, headaches and dizziness as reasons for early discontinuation of Implanon NXT. The side-effects reported in our study are consistent with the expected side-effects of Implanon NXT. We also noted that most women ( $n=93 ; 78.2 \%)$ recalled being counselled on side-effects prior to implant insertion, which might have contributed to a longer duration of Implanon NXT use.

A very small number of women had requested Implanon NXT removal for reasons other than side-effects. One participant requested removal of the implant owing to the interaction between liver-inducing medication and the implant, resulting in reduced efficacy. This is in contrast to the study done in East London, SA, where 24 of 188 (12.8\%) implants were removed owing to women being on ARVs. ${ }^{[18]}$ In the SA context, the interaction between efavirenz (EFV)-containing ARV regimens and Implanon NXT is important due to the high prevalence of HIV and the use of EFV in first-line ART regimens. The use of progestogen-only implants in women living with HIV and being administered ARVs is a World Health Organization Medical Eligibility for Contraception (MEC) category $2 .{ }^{[4]}$

Two women reported pregnancy as a reason for requesting removal of Implanon NXT. One of these women was on the implant beyond the indicated 3-year duration of use (3 years and 10 months) and her timing of conception was calculated as $\sim 6$ months after the implant had reached its duration of action. This is an interesting finding, as recent evidence suggests that etonogestrel-releasing implants are highly effective for an additional 2 years, i.e. up to 5 years. ${ }^{[22]}$ The other woman was still awaiting confirmation of pregnancy by ultrasound at the time of her interview, but on follow-up she was confirmed to be pregnant. This low failure rate supports the high efficacy of Implanon NXT as a contraceptive method. ${ }^{[3]}$

An unexpected finding was that all 5 women who had been on Implanon NXT for its full duration of use previously, had removed their second implant early and 4 of 5 had reported side-effects leading to removal. Since Implanon NXT was introduced in SA in 2014, there are limited data on repeat users.

A third of Implanon NXT users were young women between 18 and 24 years of age and just $<20 \%$ were nulliparous, indicating that implant use is an acceptable contraceptive method in younger women. More than half of users requesting Implanon NXT removal had had their implants inserted at other health facilities, implying that our study findings might not be unique to the clinic where the study was conducted. Contraceptive implants offer the benefit of a long duration of use and we found that the most common reasons for wanting to use Implanon NXT were the desire for a long-acting method and not wanting to return to the clinic frequently for follow-up.

Finally, we found that more than half of the women who had used Implanon NXT for its full duration chose to have it reinserted after removal, indicating satisfaction and acceptability of the method. We have shared these results with the $\mathrm{DoH}$, and we recommend that similar data be collected in other settings to inform local practice and to understand the experiences of SA women with Implanon NXT.

\section{Study limitations}

This was a cross-sectional study and women were not followed up prospectively. We only interviewed users at one clinic; therefore, the findings might not be generalisable to the larger population. There is potential selection bias, as the women who attended this clinic were from urban or peri-urban areas and the majority had at minimum completed secondary school. Staff at the clinic are involved in contraceptive teaching and training and might therefore be more experienced in managing side-effects and other problems, resulting in a longer duration of Implanon NXT use than at other facilities.

\section{Conclusions}

Overall, our study findings indicate that three-quarters of women requested removal of Implanon NXT because it had reached its intended 3-year duration.

Early removals were mainly due to side-effects. We also found that more than half of the women had Implanon NXT inserted again after having used it for 3 years. It is a highly effective, safe, acceptable, longacting contraceptive method and important in the SA contraceptive method mix.

Despite declining rates of insertions and concerns about early implant removals, our results are reassuring and support the use of Implanon NXT as a highly effective, long-acting, safe contraceptive method. Most early removals were due to side-effects, highlighting the importance of counselling and effective management, especially of problematic bleeding. It is important that healthcare workers who provide Implanon NXT are adequately trained and experienced in managing side-effects. Our results should also be used to strengthen programming and reproductive health service delivery. Future research is needed on the experiences of women who have Implanon NXT reinserted after removal. Further studies using larger sample sizes and across multiple sites could provide valuable insight regarding experiences of women with Implanon NXT.

\section{Declaration. None.}

Acknowledgements. We thank Nonhlanhla Mphili and Sithembile Chonco for their time and effort assisting with data collection; clinic staff for assisting with referring Implanon NXT users who requested removal; the KZN Department of Maternal, Child and Women's Health for working with us; and, finally, all the women for giving their time and providing us with valuable information.

Author contributions. IB, VM, JS and MB were responsible for conceptualisation and study design; IB was responsible for the initial draft of the protocol, study documents and the article, and was supported by JS, MB, MP, VM and SE; JS and MB, the senior authors, provided oversight and review.

Funding. MatCH Research Unit, Durban, SA.

Conflicts of interest. None.

1. Merck Sharp \& Dohme BV. Prescribing information: Implanon". https://www.merck.com/product/ usa/pi_circulars/i/implanon/implanon_pi.pdf (accessed 9 June 2018).

2. The single-rod contraceptive implant: History of subdermal contraceptive implants. http://www.arhp. 2. The single-rod contraceptive implant: History of subdermal contraceptive implants. http://www.arhp.
org/publications-and-resources/clinical-proceedings/Single-Rod/History (accessed 10 June 2018).

org/publications-and-resources/clinical-proceedings/Single-Rod/History (accessed 10 June 2018).
3S Food and Drug Administration. Drug approval package - Implanon (etonogestrel implant) 68 mg. 2009. https://www.accessdata.fda.gov/drugsatfda_docs/nda/2006/021529_implanon_toc.cfm $\mathrm{mg} .2009$. https://ww
(accessed 10 June 2018).

4. World Health Organization. WHO statement on progestogen-only implants. 2015. http://apps.who. int/iris/bitstream/handle/10665/190063/WHO_RHR_15.20_eng.pdf;jsessionid=2D9EE5268FE76092 2B29E5FB00E1A274? sequence=1 (accessed 10 June 2018)

5. Statistics South Africa. South Africa Demographic and Health Survey. Key Indicators Report. 2016 https://www.dhsprogram.com/pubs/pdf/PR84/PR84.pdf (accessed 11 June 2018).

6. National Department of Health. National Contraception and Fertility Planning Policy and Service Delivery Guidelines: A Companion to the National Contraception Clinical Guidelines. Pretoria: National Department of Health, 2012.

7ealth Systems Trust. Maternal, Child and Women's Health Barometer, 2016. Westville: Health Systems Trust, 2018

8. Makatini V, Beksinska M, Smit J. Introduction of the implant in South Africa: Impact on the contraceptive method mix and a Interntive method Internations: gatesinstitute.org/international-conference-family-planning ictp (accessed 29 August 2019).

out. Bhekisisa. https:// bhekisisa.org/article/2018-01-09-00-why-south-africa-wasnt-ready-for-the-contraceptive-implantimplanon-nxt (accessed 30 November 2018). 
10. Did Implant kill my baby? Mom fell pregnant despite taking contraceptive. 2018. https://www pressreader.com/south-africa/post/20180801/281479277222454 (accessed 30 November 2018).

11. Woman goes through hell with Implanon birth control implant. Health24. 2016. https://www.health24 com/Lifestyle/Woman/Menstruation/woman-goes-through-hell-with-implanon-birth-control$\mathrm{com} /$ Lifestyle/Woman/Menstruation/woman-gos-
implant-20160422 (accessed 30 November 2018).

12. National Department of Health. Changes in prescription of subdermal implants (Implanon) in women who are taking enzyme inducing drugs such as efavirenz for HIV, rifampicin for TB and certain drug used for epilepsy (carbamazepine, phenytoin and phenobarbital). 2014. https://sahivsoc.org/Files/ Circular\%20-\%20Changes\%20in\%20the\%20Prescription\%20of\%20Progestin\%20Subdermal\%20 Implants.pdf (accessed 30 December 2018)

13. Panday M. The contraceptive implant in South Africa - four years on. The experience of a family planning department in KwaZulu-Natal. O\&G Forum 2018;(3):18-22.

14. Blumenthal PD, Gemzell-Danielsson K, Marintcheva-Petrova M. Tolerability and clinical safety of Implanon. Eur I Contracept Reprod Health Care 2008:13(S1):29-36, https://do org $/ 10.1080 / 13625180801960012$

15. Funk S, Miller MM, Mishell DR, et al. Safety and efficacy of Implanon";, a single-rod implantable contraceptive containing etonogestrel. Contraception 2005;71(5):319-326. https://doi.org/10.1016\%2Fj. contraception.2004.11.007

16. Mansour D, Korver T, Marintcheva-Petrova $M$, et al. The effects of Implanon ${ }^{\circ}$ on menstrua bleeding patterns. Eur J Contracept Reprod Health Care 2008;13(S1):13-28. https://doi. org $/ 10.1080 \% 2 \mathrm{~F} 13625180801959931$

17. Bahamondes L, Brache V, Meirik O, et al. A 3-year multicentre randomized controlled trial of etonogestreland levonorgestrel-releasing contraceptive implants, with non-randomized matched copper-intrauterin device controls. Hum Reprod 2015;30(11):2527-2538. https://doi.org/10.1093\%2Fhumrep\%2Fdev221
18. Mrwebi KP, Goon DT, Owolabi EO, et al. Reasons for discontinuation of Implanon among users in Buffalo City Metropolitan Municipality, South Africa: A cross-sectional study. Afr J Reprod Health 2018:22(221):113-119. https://doi.org/10.29063/ajrh2018/v22i1.11

19. Pillay D, Chersich MF, Morroni C, et al. User perspectives on Implanon NXT in South Africa: A survey of 12 public-sector facilities. S Afr Med J 2017;107(10):815. https://doi.org/10.7196/ samj.2017.v107i10.1283

20. Adeagbo OA, Mullick S, Pillay D, et al. Uptake and early removals of implanon NXT in South Africa: Perceptions and attitudes of healthcare workers. S Afr Med J 2017;107(10):822-826. https://doi. org/10.7196/samj.2017.v107i10.12821

21. StataCorp. Stata Statistical Software: Release 14. 2015. http://www.stata.com (accessed 21 August 2019)

22. Ali M, Bahamondes L, Landoulsi SB. Extended effectiveness of the etonogestrel-releasing contraceptive implant and the $20 \mu \mathrm{g}$ levonorgestrel-releasing intrauterine system for 2 years beyond US Food and Drug Administration Product Labeling. Glob Health Sci Pract 2017;5(4):534-539. https://doi.org/10.9745/GHSP-D-17-00296

Accepted 28 March 2019 